\title{
Correspondence
}

\section{Single consultant posts in mental handicap}

\section{DeAR SIRS}

The article which you published from Dr Nwulu concerning his single consultant post at Rotherham (Bulletin, July 1988, 12, 279-281), was discussed by the Section for the Psychiatry of Mental Handicap at its recent meeting.

The general opinion expressed by the Committee was that although some such posts did exist, these were fortunately in the minority. Since the publication of the Normansfield Report in 1978 which highlighted the problem of single consultant posts, it has been felt that such appointments are potentially detrimental to the incumbent for some of the difficulties outlined in Dr Nwulu's article. Unfortunately, with the development of districts with small populations, the existence of such posts is likely to continue.

The Section would be very interested to know the extent of the problem and to see if some positive action could be taken in cases such as Dr Nwulu. I would therefore like to hear personally from any consultant in mental handicap who feels that they are working in isolation and under the difficulties experienced by him. This would enable the Section to debate what action could be taken by us to support this group.

Chairman-Section for the Psychiatry of Mental Handicap

Rampton Hospital

Retford

Notts

\section{The loneliness of the on-call psychiatrist: ways of easing the burden}

\section{DeAR SiRS}

There have been a number of articles published recently about stress levels and psychiatric morbidity among junior doctors. ${ }^{\prime}$ For the resident on-call psychiatrist there is an added stress, that of loneliness. In a large psychiatric hospital the duty psychiatrist is often the only doctor present on site out of hours and at weekends. As a result there is little opportunity for supportive interaction with colleagues.
Weekly Balint-type groups could provide a useful framework for discussing some of these problems and perhaps releasing some of the frustration but they are usually primarily related to patient management.

In my experience, a valuable aid to easing the burden of loneliness is through written communication in the form of an on-call diary. The diary in which I was involved started off as a method of recording the number of night calls but soon changed to a more general means of communication to describe interesting and unusual events and occasional frustrations. Reading the diary and composing entries provided an important medium for sharing difficulties and other feelings with colleagues. I can remember on occasions actually looking forward to being on call in order to read the previous week's entries. It produced a feeling that one was not alone.

I would recommend the use of an on-call diary but would point out that to be successful the majority of junior doctors would need to contribute and also the entries would need to be confidential among the junior doctor group. The diary with which I was involved lasted for about three years but unfortunately ended when only a minority of on-call doctors provided entries.

\section{High Royds Hospital}

ROBERT D. ADAMS

\section{Menston, Ilkley}

West Yorkshire

\section{References}

${ }^{1}$ FirTH-Cozens, J. (1987) Emotional distress in junior house officers. British Medical Journal, 295, 533-536.

\section{The doctrine of specific consent and research}

DeAR Sirs

Paragraph 13 of the General Medical Council statement on testing for HIV (quoted in Simmons, 1988) ${ }^{1}$ has potentially disastrous consequences for psychiatric research. It unequivocally states that specific consent is required for each and every blood investigation carried out, and that the responsibility for ensuring that this is the case lies with the performer of the tests.

In the case of patients who are considered unable to give informed consent, the general approach of 
ethical committees when approving research proposals has been to permit nearest relatives to give consent in the patient's place. This solution is no longer viable as, by logical extension, a nearest relative would be able to consent to an HIV test on the patient. In the light of this statement, rather than face a General Medical Council hearing, we have just abandoned a study into a potential biological marker for psychosis.

We are concerned that this provision for blood tests may also apply to neuro-imaging and possibly to the application of structured interviews (as the latter may also reveal unsuspected, untreatable pathology, which currently seems to be one of the cardinal problems in HIV testing.) If this is the case, it is hard to see how significant research can be conducted on any but the worried well. One of us has written previously on the specifically psychiatric problems posed by HIV $^{2,3,4}$ and it would seem that the stagnation in research suggested in the last of these may become a reality if the present doctrine of specific consent is adhered to. Given the, in our opinion, justified importance attached by the College to research as part of training, we believe that the time has come for a consensus statement on the issue of consent. Backed by this, at least some research on our more severely ill patients may be able to proceed with the investigators secure in the knowledge that, if called before the Courts to justify their actions, they can be protected by the Bolam Test in that "a body of responsible medical opinion" has sanctioned their acts - at the moment, all research on patients whose judgement is impaired places the investigator at risk of litigation.

Moorhaven Hospital

D. R. DAVIES

Ivybridge, S. Devon

Wonford House Hospital

J. C. RIGBY

Exeter, Devon

\section{References}

${ }^{1}$ Simmons, N. A. (1988) Consensus on HIV testing. British Medical Journal, 297, 356-357.

${ }^{2}$ DAVIES, D. R. (1987) HIV and the psychiatric hospital. British Journal of Psychiatry, 150, 881-882.

3 _ (1988a) Behaviourally disturbed HIV patients. British Journal of Psychiatry, 152, 577-578.

4 - (1988b) Screening for HIV. British Journal of Psychiatry, 152, 857.

\section{Mixed marriage and stress}

\section{DeAR Sirs}

In Britain today there are more than two million immigrants from the new Commonwealth and
Pakistan. There is little doubt about the multi-racial status of present British society and the resulting enculturation. I have seen both in adult and child psychiatry the increased vulnerability of an individual to a psychological breakdown because of the complex dynamics of a mixed marriage. Such a marriage has to resolve a variety of personal, social, political and cultural conflicts if it is to succeed and create a healthy family. It is further complicated by the addition of children who have to grow up at times in an immense state of confusion about their identity and sense of belonging.

Birmingham has a high concentration of ethnic population and therefore one has more opportunity to see the problems associated with mixed marriages. I feel quite strongly about a role for preventive psychiatry in this field, and the increasing need for a counselling service for this particular population. I am at present conducting a survey of mixed marriage couples and hope to be able to publish the results soon to emphasise the need for a specialised counselling service. I would welcome the views of my colleagues on the subject and of any problems they might have encountered with this population in their clinical practice.

\section{The Midland Nerve Hospital \\ Elvetham Road, Edgbaston \\ Birmingham B15 2NJ}

A. JAWAD SHEIKH

\section{Bipolar affective disorder and child psychiatry}

DEAR SIRS

The predominant theme of the Residential Meeting in the Section of Child and Adolescent Psychiatry was childhood depression and bipolar affective disorder. As a relatively inexperienced diagnostician am I mistaken in detecting signs of bipolar affective disorder within the Section itself?

In the depressive phase, the pervasive feeling of hopelessness and helplessness in response to threats and cuts in child and adolescent psychiatry is experienced; in the manic phase, distressingly raucous and ribald laughter that was provoked by Dr Skuse's video recordings of 'abnormal feeding patterns'.

What is there to learn from the Section Meeting about the management of this condition? Should I follow Professor Taylor's example and initiate intensive investigations to reveal an underlying organic pathology? Or take Professor Graham's approach and try and understand these reactions as an understandable response to everyday suffering?

We practise in difficult times; like Dr Skuse's families it seems that we regard deprivation and decay as 